\title{
The Challenge of Improving IVF Results in Normogonadotrophic (Unexpected) Young Poor Ovarian Responders: The Predictive Value of a Flexible Treatment Protocol Based on the "Biophysical Profile of the Uterus"
}

\author{
Menelaos J. Tzafetas ${ }^{1,2^{*}}$, Theoharis Tantanasis ${ }^{1}$, Vassiliki Zournatzi ${ }^{1}$, \\ Konstantinos Lathouras ${ }^{1}$, Aristotle Loufopoulos ${ }^{1,2}$ \\ ${ }^{1} \mathrm{~B}^{\prime}$ Department of Obstetrics and Gynecology, Aristotle University, Thessaloniki, Greece \\ ${ }^{2}$ IVF and Infertility Centre, Interbalcan European Hospital, Thessaloniki, Greece \\ Email: $\underline{\text { tzafetas@the.forthnet.gr, menelaos.tzafetas@gmail.com }}$
}

Received 21 August 2015; accepted 22 September 2015; published 25 September 2015

Copyright (C) 2015 by authors and Scientific Research Publishing Inc.

This work is licensed under the Creative Commons Attribution International License (CC BY). http://creativecommons.org/licenses/by/4.0/

(c) (i) Open Access

\section{Abstract}

Objective: To study whether the unexpected poor ovarian responders optimization of uterine receptivity with a flexible controlled ovarian hyper stimulation protocol based on the Biophysical Profile of the Uterus, has an impact on their reproductive performance. Design: Observational Prospective study. Setting(s): i) General hospital-IVF and Infertility Centre; ii) University hospital. Patient(s): 44 normogonadotrophic young women (26 - 38 yrs) with previous "unexpected" poor ovarian response underwent IVF/ICSI treatment on a protocol based on the Biophysical Profile of their uterus (Group A). The same patients were used as controls in a preceded IVF cycle on the conventional stimulation protocol. Intervention(s): None. Main outcome measure(s): Pregnancy, miscarriage and home take baby rates, amount and duration of gonadotropins required, number and quality of embryos resulted, Biophysical Profile of the Uterus score. Result(s). Treatment in Group A in comparison to Group B resulted in significantly larger number of eggs retrieved per patient, and improved fertilization rates and higher number of embryos/ET $(p=0.011,0.010$ and 0.034 respectively). Group $A$ also demonstrated a trend for higher rates of clinical pregnancy (29.5\% v.s. $15.9 \%)$, viable stage pregnancies $\geq 24$ weeks $(33.3 \%$ v.s. $20 \%)$ and home take babies $(26.6 \%$ v.s. $16 \%$ ). The amount of gonadotropins used per patient (IU) was similar in the two groups ( $p=$ 
0.264). Cancellation, implantation and miscarriage rates as well as embryos quality, although superior in the treatment Group A, showed no significant difference. The number of pregnancies achieved in Group A, were directly related with the score in the Biophysical Profile of the Uterus 12 point scale. Conclusion(s): Unexpected Poor Ovarian Responders on the flexible IVF/ICSI protocol (Group A), adjusting the management according to the Biophysical Profile of their uterus (duration of stimulation, day of HCG and day of embryo transfer), had a significantly better performance in comparison to the Group B managed on the conventional protocol in this difficult to manage and so far, rather understudied population.

\section{Keywords}

Normo-Gonadotrophic Unexpected Young Poor Ovarian Responders, Biophysical Profile of the Uterus, Ovarian Stimulation, in Vitro Fertilization

\section{Introduction}

Despite the enormous number of studies on poor ovarian responders published in the last 30 years, their management remains one of the biggest challenges and most controversial topics in Assisted Reproduction with less than satisfactory progress. According to recent reviews, the incidence of this condition, which also has a genetic predisposition, ranges between $9 \%$ and $24 \%$ [1]. A rate of $16.16 \%$ has been reported for "unexpected" Poor Ovarian Responders (PORs) [2]. There is no universally accepted definition, but most people refer to cases with less than 4 follicles and/or estradiol (E2) levels less than $500 \mathrm{pg} / \mathrm{ml}$ on the day of HCG administration. According to the ESHRE consensus in a first real attempt to find a common definition of "poor responders" [3], early detection of the problem with different methods of assessment of ovarian reserves, is of particular importance in order to select the most appropriate method of treatment. Today, the AFC (Andral Follicle Count) or AMH (Anti-Mullerian Hormone) measurement is recommended to predict ovarian response [4] [5], with the latter presenting as an excellent and equally promising test [6] [7]. Moreover, time interval up to 12 months does not seem to modify its predicting capacity [8]. For scientific purposes however, according to the 2011 ESHRE consensus ${ }^{3}$, more than one criterion should be contemporaneously presented.

“Unexpectedly poor responders” (unexplained) represent a heterogeneous group of patients. Different mechanisms have been proposed to explain poor ovarian response such as decreased number of FSH receptors in granulosa cells [9], defective signal transduction after FSH-receptor binding [10], anti-FSH IgA and IgG potentially exerting a local FSH antagonizing effect in maturing follicle [11], the presence of a specific FSH receptor-binding inhibitor in the follicular fluid [12] and possibly underlying occult theca cells deficiency [13]. Detailed definition of risk factors other than age has not been addressed in the 2011 ESHRE consensus on PORs. As underlined by Younis [14], "some are well established, some are still controversial and some novel candidates may be identified in the near future". A revised and updated evaluation of the risk factors, in accordance with the current knowledge and evidence, as stated by Ferraretti and Gianaroli [15], may be needed in the near future.

Uterine factor infertility is multifactorial. Although it has been intensely investigated, it is still ascribed as "the last untreatable form of female infertility", hence, the indication for transplantation of uterus with successful outcome, as reported recently [16].

Morphological appearance of the endometrium. The endometrium is one of the most dynamic structures in the body. Despite their anatomical, physiological and reproductive diversities, the mammals have an impressive synchronization between embryo and endometrial maturation during the "implantation window" (IW) of vital importance. This, to a major degree, is hormonal dependent, but also the result of an "embryo-maternal dialogue" at a systematic and molecular level [17]. High-resolution ultrasonography serves as a non-invasive method in assessing endometrial receptivity based on its pattern and thickness or, more accurately, on its volume measured by 3D/4D Ultrasound. Endometrial volume $2.5 \mathrm{ml}$ or less is associated with significantly lower endometrial vascularity and consequently, receptivity [18]. The predictive value of endometrial thickness alone, in relation to its receptivity however, appears controversial and lacking specificity [19]. Much less attention has been paid as 
a routine, on the importance of its changing morphology during the cycle. This is expressed by its evolving fine structure during the menstrual cycle and the degree of the echogenicity determined by the changes in the vascular and glandular elements, mainly by the secretions contained which reflect the degree of secretory transformation and preparedness for implantation. It has been demonstrated that echogenic patterns of the endometrium reflect histologic processes related to receptivity [20].

Blood flow impedance of the uterus. Main causes of IVF failures are poor egg quality and poor endometrial receptivity, both related to a common etiology: the impaired follicular and endometrial blood supply. Continuous and steadily increasing blood flow in the follicular wall is hormone dependent (E2, LH, HCG) and essential for the development and release of mature eggs for the formation of a receptive endometrium and the normal function of the Corpus Luteum (LDL, P). The blood supply to the endometrium is derived from branches of the uterine arteries. Emanating from the arcuate arteries (vide supra) are the radial arteries. These vessels run through the myometrium to just outside the endometrium where they form terminal branches of two types: straight and coiled. The straight branches, also known as the basal arteries, supply the basalis layer of the endometrium. The coiled branches, also known as the spiral arteries, traverse the endometrium and supply the functionalis layer. The endometrium and the spiral arteries, unlike the basal arteries, are responsive to the hormonal changes of the menstrual cycle and undergo cyclical transformations. When the spiral arterioles are inadequately developed the chances for a normal implantation may be reduced [21].

The introduction of Color Doppler processing in trans-vaginal probes apart from the anatomical image of the internal genital organs, has provided means of assessing their function as this is expressed by their blood flow changes in the reproductive organs, which vary considerably during the menstrual cycle, and enable us to depict functional abnormalities directly related to the reproductive status of the woman and not only. It was Rajat Goswamy and Patrick Steptoe who first reported that < poor mid secretory uterine artery response expressed as absence of qualitative changes in diastolic flow velocity were indicative of poor uterine perfusion>, and was considered a cause of infertility [22]. An observation confirmed histologically following endometrial biopsies [23]-[25].

In the conventional Controlled Ovarian Hyperstimulation (c-COH: at least 2 follicles of $\geq 16$ mm diameter and E2 levels of $150-200 \mathrm{pgr} / \mathrm{ml}$ per follicle) one of the main criteria before triggering ovulation has been the size of the follicles, which doesn't necessarily reflect the stage of its maturity and the degree of endometrial receptivity. As a simple example we refer to follicles stimulated with Clomiphene Citrate where their size tends to be disproportionately larger in relation to the utero-ovarian blood flow impedance (PI, RI), a parameter with higher predictive value [26] [27]. It was demonstrated that progressive decrease in the pulsatility index (PI) of the uterine arteries during the second half of the menstrual cycle was associated with successful IVF pregnancies [28]. Further studies reported that the uterus is likely to be unreceptive when uterine artery PI is $\geq 3$ and resistance in$\operatorname{dex}(\mathrm{RI} \geq 0.95)[29]-[32]$

Vascularity of the uterus. Myometrial and endometrial perfusion is directly related to the aetiology of uterine factor infertility. Using dynamic methods like transvaginal color and power flow Doppler images as well as 3-D/4-D ultrasound, it has been demonstrated that detectable myometrial blood flow was associated with higher implantation and clinical pregnancy rates [33]-[36]. The same applies for the endometrium although its prognostic value remains somehow controversial. The parameters mentioned above, not always adequately synchronized, have been extensively studied and analyzed but rather independently and, not infrequently, based on a single measurement [37] [38]. Triggering ovulation too early or too late reduces success rates. The selection of a more optimal day for HCG administration is of paramount importance and this is more likely when based on a combined evaluation of all parameters related with uterine receptivity.

Since the publication of the Bologna criteria in 2011, aspects of them have been cited several times, whilst others have been criticized. It has been suggested for instance that, patients with "advanced age" or any other known risk factor i.e. ovarian surgery for endometriosis or other pathology, should not be classified as PORs according to the above criteria, but more properly be defined as “expected poor responders", instead [14] [15].

The present study focuses on a selected, rare group of normo-gonadotrophic young PORs under 38 yrs (34.0 \pm 4.2) with no evidence of premature menopause, as included in the afore mentioned criteria. The purpose of the study was to study whether, in addition to the criteria used in the c- $\mathrm{COH}$, modification of the management (duration of $\mathrm{COH}$, day of HCG injection, day of egg collection and embryo transfer-ET) based on the Biophysical profile of the uterus (BPU) (Table 1), improves the IVF/ICSI outcome in these, difficult to manage, "unexplained” PORs. Unlike previous reports, contemporaneous and repeat evaluation of all BPU variables related to uterine receptivity was monitored (Table 1). 
Table 1. The Biophysical Profile of the Uterus (BPU 12 point scale).

\begin{tabular}{ll}
\hline Variable & Score \\
\hline 1) Endometrial thickness $(\mathbf{a}, \mathbf{b}, \mathbf{c})$ & \\
Thickness a: $<7 \mathrm{~mm}$ & 1 point, \\
b: $7-10 \mathrm{~mm}$ & 2 points \\
c: $\geq 11 \mathrm{~mm}$ & 3 points \\
\hline
\end{tabular}

\section{2) Endometrial morphology}

Type A: immature trilaminar endometrium with three distinct zones of
hyperechoic outer and inner layer and hypo-echoic dark parenchyma*
2 points
Type B: preovulatory trilaminar endometrium, with iso-echoic parenchyma* 3 points
Type C: over-mature, hyper-echoic endometrium with loss of the three zone structure
1 point

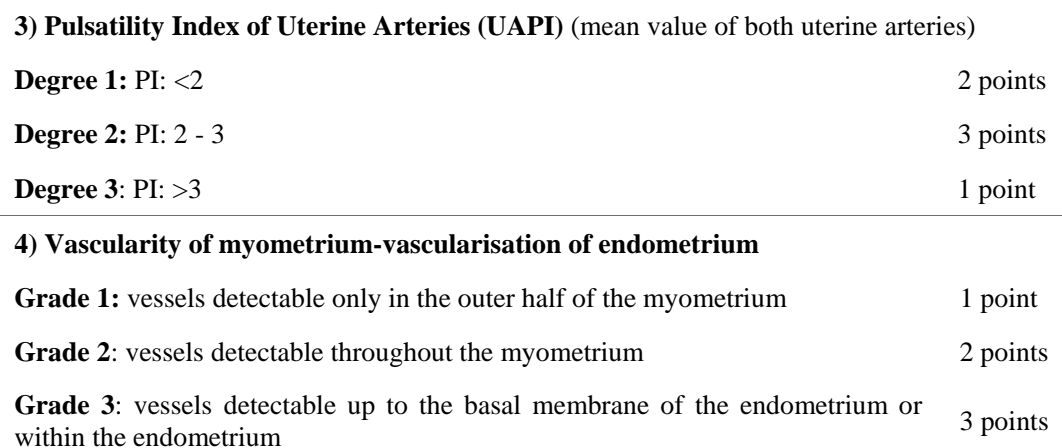

within the endometrium

*in comparison to the myometrium.

\section{Materials and Methods}

A total of 44 normogonadotrophic young infertile women with a previous history of "unexpected" (unexplained) poor ovarian response were enrolled in a prospective observational study (Group A). The selection criteria used were: specific group of infertile women $\leq 38$ years of age $(34.0 \pm 4.2)$ with poor ovarian response despite their normal hormonal profile (normogonadotrophic). The duration of stimulation, the day of HCG administration, egg collection and day of embryo transfer, were mainly determined by the uterine receptivity score of a 12 point scale based on the combined assessment of the BPU variables individually (Table 1). With the aim to achieve the most optimal uterine receptivity conditions prior to ET, a flexible protocol was followed (Figure 1). If, on the day of HCG administration, according to the c-COH, the BPU total score was not satisfactory (cut off point $\leq 6$ ), the stimulation was prolonged for 24 to 72 hrs before triggering ovulation, with a daily hormonal (E2, LH) and sonographic re-assessment of the BPU variables in an attempt to improve them further. The same applied for the ET, which again varied accordingly between day 2 and 5 in an attempt to coincide at the best possible with the implantation window (IW) as this was indicated by the BPU indices. The aim was to achieve a preovulatory endometrium (Type 2), PI between 2 and 3, good vascularity myometrium (Grade 2 - 3) preferably associated with detectable sub and endometrial vascularization (Table 1). At the presence of an over-mature endometrium on the day of egg collection (about to lose its trilaminar structure), early ET was preferred (on Day 2) or embryo freezing was decided in cases with complete loss of the three line structure endometrium-invariably incompatible with implantation (over-mature Type 3).

A General Electric Voluson E8 ultrasound machine was used by a single examiner for the sonographic evaluation of the uterine receptivity based on the BPU variables. Blood sample was taken on the $2^{\text {nd }}$ day of the cycle for E2 and progesterone (P) followed by 150 - 300 IU/day gonadotropin stimulation from day 3 of the menstrual cycle (rec-FSH/HMG). In view of their previous history of unexpected POR Group A patients were all treated with GnRH antagonist protocol (ganirelix $0.25 \mathrm{mg} / 0.5 \mathrm{ml}$, Orgalutran/INN-Cetrolelix $0.25 \mathrm{mg}$ Cetrotite, Europa). 


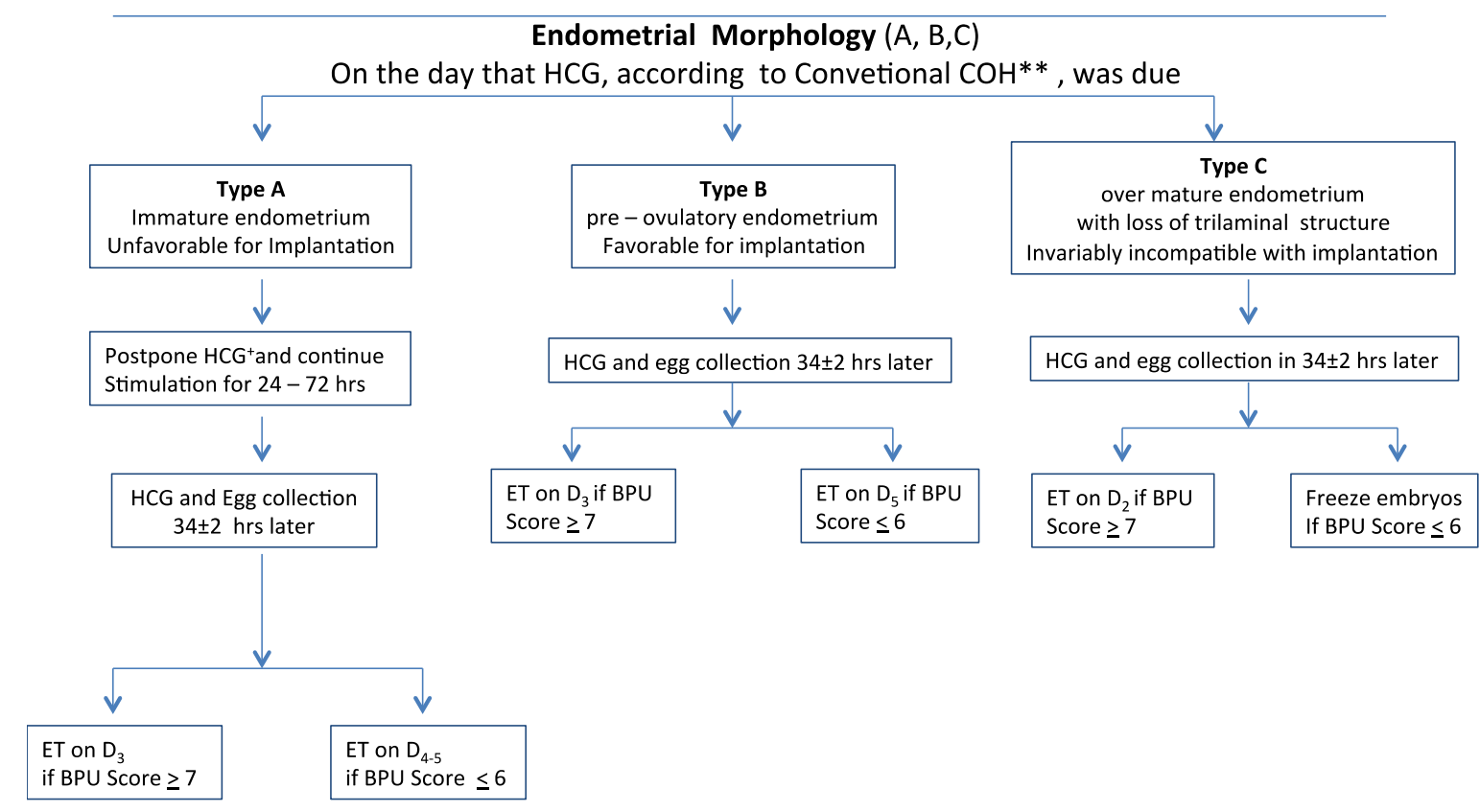

Figure 1. Proposed IVF/ ICSI management based on the Biophysical Profile of the uterus* (BPU 12 point scale). *As in Table 1; ** >2 Follicles, >16 mm diameter and E2: 150 - 200/follicle.

The same patients were used as controls $(n=44)$ in a preceded IVF cycle stimulated with the conventional $\mathrm{COH}$ protocol (c-COH): $\geq 2$ follicles, $\geq 16 \mathrm{~mm}$ in size, 150 - $200 \mathrm{pg} / \mathrm{ml}$ per follicle) (Group B).

\section{Statistics}

All data were analyzed with the statistical package SPSS (version 17.0; SPSS, Chicago, IL, USA). Data are presented as percentages for categorical variables and as mean and standard deviation for continuous variables. Differences in categorical and continuous variables between groups were assessed with the $\chi^{2}$ test and the independent samples t-test, respectively. In all cases, a two-tailed $\mathrm{p}<0.05$ was considered significant.

\section{Results}

Table 2 shows the clinical characteristics and IVF/ICSI-ET performance in the two treatment cycles (Groups A and B). The indications for IVF/ICSI remained the same in both groups: Unexplained infertility (40.90\%), male (29.54\%), tubal (13.63\%) and others (15.9\%). There was no significant difference in the total amount of gonadotropins required, the peak Estradiol levels (E2) reached, the cancellation and implantation rates. In view of the previous history of unexpected POR, all Group A patients (BPU protocol) underwent ICSI procedure, except 2 with TESE/ICSI. In Group B (preceded cycle on the c-COH protocol within an interim period of 6.5 and 8 months), 12 had classical IVF, 28 ICSI, and 2 TESE/ICSI treatment (the same ones as in Group A). Due to the relatively short interim period between the two treatment cycles (4 - 9 months) basal hormonal profile was as per Group B: basal FSH and LH levels within normal limits $(8.8 \pm 2.6 \mathrm{mIU} / \mathrm{ml}$ and $6.7 \pm 2.3 \mathrm{mIU} / \mathrm{ml}$, respectively) and Anti-Müllerian hormone (AMH) varied between 0.6 and $21.8 \mathrm{ng} / \mathrm{ml}(7.0 \pm 6.1 \mathrm{ng} / \mathrm{ml})$.

In the present study, treatment based on the biophysical profile of the uterus (Group A) increased the number of oocytes retrieved per patient, improved fertilization rates and number of embryos transferred per ET ( $\mathrm{p}=$ 0.011, 0.01 and 0.034 respectively), although embryo quality was similar in both treatment cycles (Grade A embryos: $\mathrm{p}=0.285$ ). There was also a definite trend for higher rates of clinical pregnancy, viable stage pregnancy ( $\geq 24$ weeks) and home take babies, although these differences did not reach significance (Table 2).

In Group A, clinical pregnancies increased parallel to the BPU score (Figure 2). Women with BPU score 11 - 


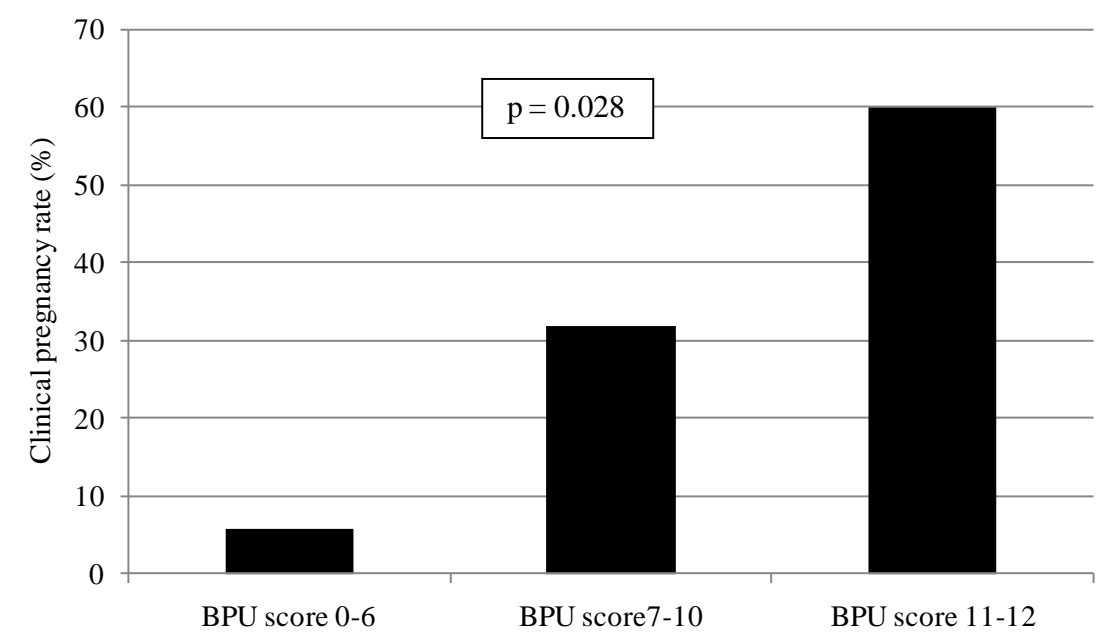

Figure 2. Clinical pregnancy rates according to BPU score categories. In the study group (A), there was a marked tendency for clinical pregnancies to increase parallel to the BPU score (12 point scale).

Table 2. IVF/ICSI results in normo-gonadotrophic young poor responders (unexpected PORs).

\begin{tabular}{|c|c|c|c|}
\hline & Group A (Study Group)* & Group B (Control Group)** & $\mathrm{p}$ \\
\hline Number of cases & 44 & 44 & \\
\hline Age (years) & $34.0 \pm 4.2$ & $33.5 \pm 3.9$ & 0.531 \\
\hline Body mass index $\left(\mathrm{kg} / \mathrm{m}^{2}\right)$ & $22.9 \pm 2.8$ & $22.4 \pm 2.6$ & 0.403 \\
\hline Infertility duration (years) & $5.0 \pm 2.9$ & $4.6 \pm 3.3$ & 0.586 \\
\hline Previous IVF attempts & $2.4 \pm 1.2$ & $1.4 \pm 1.1$ & $<0.001$ \\
\hline E2 on HCG day (pg/ml) & $573.5 \pm 363.9$ & $451.8 \pm 238.4$ & 0.068 \\
\hline Gonadotropins used per patient (IU) & $2704.9 \pm 501.2$ & $2580.1 \pm 538.2$ & 0.264 \\
\hline Oocytes retrieved per patient & $2.3 \pm 1.2$ & $1.7 \pm 0.9$ & 0.011 \\
\hline Fertilization rate & $67.3 \%(68 / 101)$ & $46.6 \%(34 / 73)$ & 0.01 \\
\hline Cancellation rate & $31.8 \%(14 / 44)$ & $40.9 \%(18 / 44)$ & 0.506 \\
\hline Number of embryos transferred (total) & 50 & 34 & \\
\hline Patients with embryo transfer (ET) & $68.2 \%(30 / 44)$ & $54.5 \%(25 / 44)$ & 0.274 \\
\hline Number of embryos/embryo transfer (ET) & $1.7 \pm 0.5$ & $1.4 \pm 0.5$ & 0.034 \\
\hline Implantation rate & $30 \%(15 / 50)$ & $20.5 \%(7 / 34)$ & 0.478 \\
\hline \multicolumn{4}{|l|}{ Clinical pregnancies: } \\
\hline Per Cycle & $29.5 \%(13 / 44)$ & $15.9 \%(7 / 44)$ & 0.203 \\
\hline Per Embryo Transfer (ET) & $43.4 \%(13 / 30)$ & $28.0 \%(7 / 25)$ & 0.37 \\
\hline Miscarriages & $23.0 \%(3 / 13)$ & $28.5 \%(2 / 7)$ & 1,000 \\
\hline Viable pregnancies ( $>24$ wks/embryo transfer) & $33.3 \%(10 / 30)$ & 20\%(5/25) (1 NND@26 wk) & 0.423 \\
\hline Home take babies/embryo transfer & $26.6 \%(8 / 30)$ & $16 \%(4 / 25)$ & 0.531 \\
\hline
\end{tabular}

*Group A: Study group: BPU treatment protocol; **Group B: Control group: Conventional treatment protocol (c-COH $=\geq 2$ folicles, $\geq 16 \mathrm{~mm}$ in size, $150-200 \mathrm{pg} / \mathrm{ml}$ per follicle). 
12 had higher pregnancy rates than those with BPU score 7 - 10 or $\leq 6$.

\section{Discussion}

Up to $85 \%$ of the embryos replaced into the uterine cavity fail to implant [39]. This, in addition to the quality of the embryos, very much depends on the overall uterine receptivity and the timing of the embryo transfer (ET) [40] [41]. Since the introduction of Assisted Reproduction there has been a significant progress in improving ovarian stimulation, oocyte retrieval, IVF and embryo transfer. Furthermore, culturing embryos up to the blastocyst stage with the introduction of sequential culture media enabled us to select embryos more competent to implant. On the other hand, combined assessment and/or improvement of the multi variant uterine receptivity based mostly on advanced and repeat sonographic analyses, especially that of the endometrium, has not been established accordingly on a routine basis because, among others, it is time consuming and requires the involvement of specially trained and readily available sonographers.

In this study we tried to look into whether the management of young normo-gonadotropic (unexpected) infertile poor responding women (un-PORs) an uncommon and rather understudied population as already stated, improved their reproductive performance after a combined evaluation of certain uterine receptivity variables in the BPU (Table 1). A flexible protocol was deployed with the aim to achieve the optimal uterine conditions before triggering ovulation and to determine the most favourable day for embryo transfer in an attempt to identify or be close to the IW.

So far, in view of the unsatisfactory reproductive performance of this particularly challenging group of patients (un-PORs) and the recognized fact that no single regimen will benefit all PORs, a variety of regimes have been proposed.

Crosignany et al. [42] suggested that high initial doses of urinary FSH could be successfully used to achieve follicular growth in $70 \%$ of prior PORs, a view shared by others, too [43]. Similar studies though, did not confirm such a beneficial effect concluding that little benefit would be expected from raising the initial daily dose to $>450 \mathrm{IU} / \mathrm{d}$ and the options of egg donation or adoption should therefore be considered [44]. Increasing the dose of r-FSH/HMG in a second stimulation cycle after first unsuccessful attempt, it has been shown it will add only to the cost and discomfort of the treatment and might even adversely affect pregnancy rates [45]-[47]. In certain cases, however, poor response may be associated with suboptimal exposure to gonadotrophins, for example in obese women [48] or the presence of FSH receptor subtypes which render the follicles less sensitive to exogenous gonadotrophins [49].

In this study, the maximum daily dose did not exceed the above mentioned limits. A small increase in comparison to the control cycle, was due to the prolongation of the treatment in 9 cases for the reasons explained (p = 0.264; Table 2).

The reported beneficial effect of dehydro-epiandrosterone (DHEA) therapy on improvement of reproduction outcome [50]-[52] remains uncertain. A review of the previously published studies between 2000 and 2014 does not provide clear evidence in favour. Interestingly, one prospective randomized study showed that DHEA might be beneficial but in women without diminished ovarian reserve (normal responders) [53].

With regards to supplementation of growth hormone, although the success rates are still not as high and with a considerable cost involved, several studies have shown improved IVF live birth success rates in PORs [54] [55]. Since the early application of genetics into ART there have been further advances in the techniques and applications. Zech and colleagues [56] recently proposed preimplantation genetic screening (PGS) following accumulation cycles by repeated $\mathrm{COH}$ with subsequent freezing of blastocysts, as a promising new approach for low responders, especially in cases of advanced maternal age. Finally, although not universally accepted [57], patients with non-pathological ovarian reserve tests and with previous "unexpected” POR, possibly due to an underlying occult theca cells deficiency, seem to benefit from r-LH addition in subsequent cycles without the need to increase the r-FSH starting dose [13].

\section{Conclusion}

In the present study, treatment based on the biophysical profile of the uterus increased the number of oocytes retrieved per patient, and improved fertilization rates and number of embryos/ET significantly. There has been a marked tendency for clinical pregnancies to increase parallel to the score in the BPU scale in Group A. The fact that there was no significant difference in the egg quality in the two treatment cycles $(p=0.285)$ indicated that 
the improved outcome in Group A could be attributed to the increased uterine receptivity resulted from the protocol applied in this group. Furthermore, a clear trend for higher rates of clinical pregnancy, viable stage pregnancy ( $\geq 24$ weeks) and home take babies was recorded, although these differences did not reach significance. Despite their striking numerical difference, it is possible that our study has limited power to show a significant difference between the two groups due to the relatively small sample size of these rather than infrequently encountered patients with "unexpected" POR that they represent only about 1 in 6 of the total PORs [2]. Only Caucasian normogonadotropic, unexpectedly infertile women from a single centre with a rather heterogeneous etiology that had not been specifically addressed in the 2011 ESHRE consensus on PORs [3], were evaluated in this study. Therefore, larger studies are needed to confirm these promising results. The findings in this study indicate that individualized "fine tuning" of the BPU variables may improve uterine receptivity and contribute in still achieving reasonable pregnancy rates before resorting to egg donation.

\section{References}

[1] Polyzos, N.P. and Devroey, P. (2011) A Systematic Review of Randomized Trials for the Treatment of Poor Ovarian Responders: Is There Any Light at the End of the tunnel? Fertility and Sterility, 96, 1058-1061. http://dx.doi.org/10.1016/j.fertnstert.2011.09.048

[2] Wei, Z., Cheng, X., Li, H., Cao, Y., Cong, L., Zhou, P. and Li, J. (2010) Effects of Prolonging Administration Gonadotropin on Unexpectedly Poor Ovarian Responders Undergoing in Vitro Fertilization. Reproductive Biology and Endocrinology, 8, 26-34. http://dx.doi.org/10.1186/1477-7827-8-26

[3] Ferraretti, A.P., La Marca, A., Fauser, B.C., Tarlatzis, B., Nargund, G. and Gianaroli, L. (2011) ESHRE Consensus on the Definition of "Poor Response" to Ovarian Stimulation for in Vitro Fertilization: The Bologna Criteria. Human Reproduction, 26, 1616-1624. http://dx.doi.org/10.1093/humrep/der092

[4] The Practice Committee of ASRM, 2012.

[5] La Marka, A. and Sunkara, S.K. (2014) Individualization of Controlled Ovarian Stimulation in IVF Using Ovarian Reserve Markers: From Theory to Practice. Human Reproduction Update, 20, 124-140. http://dx.doi.org/10.1093/humupd/dmt037

[6] Broer, S.L., Dólleman, M., van Disseldorp, J., et al. (2013) Prediction of an Excessive Response in in Vitro Fertilization from Patient Characteristics and Ovarian Reserve Tests and Comparison in Subgroups: An Individual Patient Data Meta-Analysis. Fertility and Sterility, 100, 420-429. http://dx.doi.org/10.1016/j.fertnstert.2013.04.024

[7] Kamel, H.M., Amin, A.H. and Al-Adawy, A.R. (2014) Basal Serum Anti-Mullerian Hormone (AMH) Is a Promising Test in Prediction of Occurrence of Pregnancy Rate in Infertile Women Undergoing ICSI Cycles. Clinical Laboratory, 60, 1717-1723.

[8] Polyzos, N.P., Nelson, S.M., Stoop, D., et al. (2013) Does the Time Interval between Antimüllerian Hormone Serum Sampling and Initiation of Ovarian Stimulation Affect Its Predictive Ability in in Vitro Fertilization-Intracytoplasmic Sperm Injection Cycles with a Gonadotropin-Releasing Hormone Antagonist? A Retrospective Single-Center Study. Fertility and Sterility, 100, 438-444. http://dx.doi.org/10.1016/j.fertnstert.2013.03.031

[9] Zeleznik, A.J., Schuler, H.M. and Reichert, L.E. (1981) Gonadotropin-Binding Sites in the rhesus Monkey Ovary: Role of the Vasculature in the Selective Distribution of Human Chorionic Gonadotropin to the Preovulatory Follicle. Endocrinology, 109, 356-362. http://dx.doi.org/10.1210/endo-109-2-356

[10] Hernandez, E.R., Hurwitz, A. and Vera, A. (1992) Expression of the Genes Encoding the Insulin-Like Growth Factors and Their Receptors in the Human Ovary. Journal of Clinical Endocrinology \& Metabolism, 74, 419-425.

[11] Haller, K., Salumets, A. and Uibo, R. (2008) Anti-FSH Antibodies Associate with Poor Outcome of Ovarian Stimulation in IVF. Reproductive BioMedicine Online, 16, 350-355. http://dx.doi.org/10.1016/S1472-6483(10)60595-0

[12] Lee, D.W., Grasso, P., Dattatreyamurty, B., Deziel, M.R. and Reichert, L.E. (1993) Purification of a High Molecular Weight Follicle-Stimulating Hormone Receptor-Binding Inhibitor from Human Follicular Fluid. The Journal of Clinical Endocrinology and Metabolism, 7, 163-168.

[13] Papaleo, E., Vanni, V.S., Vigano, P., La Marca, A., Pagliardini, L., Vitrano, R., Zanirato, M., Marsiglio, E., Privitera, L., Cantiani, M. and Recombinant, L.H. (2014) Administration in Subsequent Cycle after "Unexpected"' Poor Response to Recombinant FSH Monotherapy. Gynecological Endocrinology, 11, 813-816. http://dx.doi.org/10.3109/09513590.2014.932342

[14] Younis, J.S. (2012) The Bologna Criteria for Poor Ovarian Response; Has the Job Been Accomplished? Human Reproduction, 27, 1874-1875. http://dx.doi.org/10.1093/humrep/des118

[15] Ferraretti, A.P. and Gianaroli, L. (2014) The Bologna Criteria for the Definition of Poor Ovarian Responders: Is There a Need for Revision? Human Reproduction, 9, 1842-1845. http://dx.doi.org/10.1093/humrep/deu139 
[16] Brännström, M., Johannesson, L., Bokström, L., Kvarnström, N., Mölne, J., Dahm-Kähler, P., Enskog, A., Milenkovic, M., Ekberg, J., Diaz-Garcia, C., Gäbel, M., Hanafy, A., Hagberg, H., Olausson, M. and Nilsson, L. (2015) Livebirth after Uterus Transplantation. The Lancet, 385, 607-616.

[17] Aplin, J.D. (2009) MUC-1 Glycosylation in Endometrium: Possible Roles of the Apical Glycocalyx at Implantation. Human Reproduction, 14, 17-25. http://dx.doi.org/10.1093/humrep/14.suppl_2.17

[18] Ng, E.H., Yeung, W.S. and Ho, P.C. (2009) Endometrial and Sub Endometrial Vascularity Are Significantly Lower in Patients with Endometrial Volume $2.5 \mathrm{ml}$ or Less. Reproductive BioMedicine Online, 18, 262-268. http://dx.doi.org/10.1016/S1472-6483(10)60264-7

[19] Sundstrom, P. (1998) Establishment of a Successful Pregnancy Following IVF with an Endometrial Thickness of No More Than 4 mm. Human Reproduction, 13, 1550-1552. http://dx.doi.org/10.1093/humrep/13.6.1550

[20] Dallenbach-Hellweg, G. (1981) Histopathology of the Endometrium. Springer-Verlag, Berlin, New York. http://dx.doi.org/10.1007/978-3-642-96638-5

[21] Deligdisch, L. (1991) Endometrial Response to Hormonal Therapy. In: Altchek, A. and Deligdisch, L., Eds., The Uterus, Springer-Verlag, Berlin, New York, 102-114. http://dx.doi.org/10.1007/978-1-4613-9086-2_8

[22] Goswamy, R.K., Williams, G. and Steptoe, P.C. (1988) Decreased Uterine Perfusion-A Cause of Infertility. Human Reproduction, 3, 955.

[23] Fleischer, A.C., Kalemeris, G.C., Machin, J.E., Entman, S.S. and Everett Jr., A.E. (1986) Sonographic Depiction of Normal and Abnormal Endometrium with Histopathologic Correlation. Journal of Ultrasound in Medicine, 5, 445-452.

[24] Kupesic, S., Kurjak, A., Vujisic, S. and Petrovic, Z. (1997) Luteal Phase Defect Comparison between Doppler Velocimetry, Histological and Hormonal Markers. Ultrasound in Obstetrics \& Gynecology, 9, 1-8. http://dx.doi.org/10.1046/j.1469-0705.1997.09020105.x

[25] Nikas, G., Develioglou, O.H., Toner, J.P. and Jones Jr., H.W. (1999) Endometrial Pinopodes Indicate a Shift in the Window of Receptivity in IVF Cycles. Human Reproduction, 143, 787-792. http://dx.doi.org/10.1093/humrep/14.3.787

[26] Pellicer, A., Ballester, M.J., Serrano, M.D., Mir, A., Serra, V., Remohi, J. and Musoles, F.M. (1994) Aetiological Factors Involved in the Low Response to Gonadotrophins in Infertile Women with Normal Basal Serum Follicle Stimulating Hormone Levels. Human Reproduction, 5, 806-811.

[27] Palatnik, A., Strawn, E., Szabo, A. and Robb, P. (2013) What Is the Optimal Follicular Size before Triggering Ovulation in Trauterine Insemination Cycles with Clomiphene Citrate or Letrozole? An Analysis of 988 Cycles. Fertility and Sterility, 100, 341-348.

[28] Battaglia, C., Larocca, E., Lanzanni, A., Valentini, M. and Genazzani, A.R. (1990) Doppler Ultrasound Studies of the Uterine Arteries in Spontaneous and IVF Stimulated Ovarian Cycles. Gynecological Endocrinology, 4, 245-250. http://dx.doi.org/10.3109/09513599009024978

[29] Zaidi, J., Rudiger, P., Shaker, A., Kyei Mensah, A., Stuart, C. and Tan, S.L. (1996) Assessment of Uterine Artery Blood Flow on the Day of HCG Administration by Transvaginal Color. Doppler Ultrasound in an in Vitro Fertilization Program. Fertility and Sterility, 2, 377-381.

[30] Cassiatore, B., Simberg, N., Fusato, P. and Tiitinen, A. (1996) Transvaginal Doppler Study of Uterine Artery Blood Flow in Vitro Fertilization-Embryo Transfer Cycles. Fertility and Sterility, 66, 130-134.

[31] Edi-Osagie, E.C.O., Seif, M.W. and Alpin, J.D. (2004) Characterizing the Endometrium in Unexplained and Tubal Factor Infertility: A Multiparametric Investigation. Fertility and Sterility, 82, 1379-1389. http://dx.doi.org/10.1016/j.fertnstert.2004.04.046

[32] Ozturk, O., Bhattacharya, S., Saridogan, E., Jauniaux, E. and Templeton, A. (2004) Role of Utero-Ovarian Vascular Impedance: Predictor of on Going Pregnancy in an IVF-Embryo Transfer Programme. Reproductive BioMedicine Online, 9, 299-305. http://dx.doi.org/10.1016/S1472-6483(10)62145-1

[33] Schild, R.L., Holthaus, S., d’Alquen, J., et al. (2000) Measurement of Endometrial Blood Flow Using 3D Ultrasound Is of Prognostic Value with Regard to the Implantation Rates after IVF. Human Reproduction, 15, 89-94. http://dx.doi.org/10.1093/humrep/15.1.89

[34] Wu, H.M., Chiang, C.H., Huang, H.Y., Chao, A.S., Wuang, H.S. and Soong, Y.K. (2002) Detection of Subendometrial Vascularization Flow Index by Three-Dimentional Ultrasound May Be Useful in Predicting the Pregnancy Rate for Patients Undergoing in Vitro Fertilization-Embryo Transfer. Fertility and Sterility, 79, 5007-5011.

[35] Wang, L., Qiao, J., Zhen, X. and Liu, Z. (2010) Role of Endometrial Blood Flow Assessment with Color Doppler Energy in Predicting Pregnancy Outcome of IVF-Et Cycles. Reproductive Biology and Endocrinology, 8, 122-126. http://dx.doi.org/10.1186/1477-7827-8-122

[36] Singh, N., Bahadur, A., Mittal, S., Malhotra, N. and Bhatt, A. (2011) Predictive Calue of Endometrial Thickness, Pattern and Sub-Endometrail Blood Flowson the Day of HCG by 2D Doppler in in Vitro Fertilization Cycles: A Prospec- 
tive Clinical Study from a Tertiary Cae Unit. Journal of Human Reproductive Sciences, 1, 29-33.

[37] Shrestha, S.M., Costello, M.F., Sjoblom, P., et al. (2004) The Blood Flow in the Follicular Wall Is a Good Prognostic Index for a Positive Outcome But Not That in the Endometrium. Biological Journals and Abbreviations, 21, 387-395. http://dx.doi.org/10.1007/s10815-004-7526-7

[38] Yu, N.G., et al. (2006) Single Endometrial and Sub-Endometrial Blood Flow Measurement Is Lacking Prognostic Value. Human Reproduction, 21, 164-170.

[39] Edwards, R.G. and Brody, S.A. (1995) Natural Cycle and Ovarian Stimulation in Assisted Conception. WB Saunders, Philadelphia, 233-284.

[40] Steer, C.V., Mills, C.L., Tan, S.L., Campbe, S. and Edwards, R.G. (1992) The Cumulative Embryo Score: A Predictive Embryo Scoring Technique to Select the Optimal Number of Embryos to Transfer in an in-Vitro Fertilization and Embryo Transfer Programme. Human Reproduction, 7, 117-119.

[41] Fanchin, R. (2001) Assessing Uterine Receptivity in 2001: Ultrasonographic Glances at the New Millenium. Annals of the New York Academy of Sciences, 973, 185-202. http://dx.doi.org/10.1111/j.1749-6632.2001.tb03802.x

[42] Crosignani, P., Ragni, G., Lombroso, G., Scardirelli, C., de Laurentis, L., Caccamo, A., et al. (1989) IVF: Induction of Ovulation in Poor Responders. Journal of Steroid Biochemistry, 32, 171-173. http://dx.doi.org/10.1016/0022-4731(89)90160-X

[43] Hofmann, G., Toner, J., Muasher, S. and Jones, G. (1989) High-Dose Follicle-Stimulating Hormone (FSH) Ovarian Stimulation in Low-Responder Patients for in Vitro Fertilization. Journal of in Vitro Fertilization and Embryo Transfer, 6, 285-289. http://dx.doi.org/10.1007/BF01139183

[44] Haas, J., Zilberberg, E., Machtinger, R., Kedem, A., Hourvitz, A. and Olvieto, R. (2015) Do Poor Responders Benefit from Increasing the Daily Gonadotropin Dose during Controlled Ovarian Hyperstimulation for IVF? Gynecological Endocrinology, 1, 79-82. http://dx.doi.org/10.3109/09513590.2014.959919

[45] Ben-Rafael, Z., Benadiva, C., Ansmanas, M., Barder, B., Blasco, L., Flickinger, G., et al. (1987) Dose of human menopausal gonadotropin influences the outcome of an in vitro fertilization program. Fertility and Sterility, 48, 964-968.

[46] Surrey, E.S. and Schoolcraft, W.B. (2000) Evaluating Strategies for Improving Ovarian Response of the Poor Responder Undergoing Assisted Reproductive Techniques. Fertility and Sterility, 4, 667-676. http://dx.doi.org/10.1016/S0015-0282(99)00630-5

[47] Tarlatzis, B.C., Zepiridis, L., Grimbizis, G. and Bontis, J. (2003) Clinical Management of Low Ovarian Response to Stimulation for IVF: A Systematic Review. Human Reproduction Update, 9, 61-76. http://dx.doi.org/10.1093/humupd/dmg007

[48] Maheshwari, A., Stofberg, L. and Bhattacharya, S. (2007) Effect of Overweight and Obesity on Assisted Reproductive Technology-A Systematic Review. Human Reproduction Update, 13, 433-444. http://dx.doi.org/10.1093/humupd/dmm017

[49] Simoni, M., Nieschlag, E. and Gromoll, J. (2002) Isoforms and Single Nucleotide Polymorphisms of the FSH Receptor Gene: Implications for Human Reproduction. Human Reproduction Update, 8, 413-421. http://dx.doi.org/10.1093/humupd/8.5.413

[50] Jirge, P.R., Cavali, S.M. and Bhomkar, D.A. (2014) Impact of Dehydroepiandrosterone on Clinical Outcome in Poor Responders: A Pilot Study in Women Undergoing in Citro Fertilization, Using Bologna Criteria. Journal of Human Reproductive Sciences, 7, 175-180. http://dx.doi.org/10.4103/0974-1208.142477

[51] Vlahos, N., Papalouka, M., Triantafyllidou, O., Vlahos, A., Grimbizis, G., Creatsas, G. and Zikopoulos, K. (2015) Dehydroepiandrosterone Administration before IVF in Poor Responders: A Prospective Cohort Study. Reproductive BioMedicine Online, 2, 191-196. http://dx.doi.org/10.1016/j.rbmo.2014.10.005

[52] Kolibianakis, E.M., Venetis, C.A., and Tarlatzis, B.C. (2011) DHEA Administration in Poor Responders. Human Reproduction, 26, 730-731. http://dx.doi.org/10.1093/humrep/deq397

[53] Yeung, T.W., Chai, J., Li, R.H., Lee, V.C., Ho, P.C. and Ng, E.H. (2014) A Randomized, Controlled, Pilot Trial on the Effect of Dehydroepiandrosterone on Ovarian Response Markers, Ovarian Response and in Vitro Fertilization Outcomes in Poor Responders. Fertility and Sterility, 1, 108-115. http://dx.doi.org/10.1016/j.fertnstert.2014.03.044

[54] Hsu, C.J. (1987) Concomitant Effects of Growth Hormone on Secretion of Insulin-Like Growth Factor I and Progesterone by Cultured Porcine Granulose Cells. Endocrinology, 121, 1343-1348. http://dx.doi.org/10.1210/endo-121-4-1343

[55] Kolibianakis, E.M., Venetis, C.A., Diedrich, K., Tarlatzis, B.C. and Griesinger, G. (2009) Adition of Growth Hormone to Gonadotrophins in Ovarian Stimulation of Poor Responders Treated by in-Vitro Fertilization: A Systematic Review and Meta-Analysis. Human Reproduction Update, 6, 613-622. http://dx.doi.org/10.1093/humupd/dmp026

[56] Zech, M., Baldauf, S., Comploj, G., Murtinger, M., Spitzer, M., Hradecky, L., Ajavi, R., Schuff, M. and Zech, H. 
(2015) Ovarian Stimulation in ART-Unwinding Pressing Issues. Minerva Ginecologica, 67, 127-147.

[57] Fan, W., Li, S., Chen, Q., Huang, Z., Ma, Q. and Wang, Y. (2013) Recombinant Luteinizing Hormone Supplementation in Poor Responders Undergoing IVF: A Systematic Review and Meta-Analysis. Gynecological Endocrinology, 4, 278-284. http://dx.doi.org/10.3109/09513590.2012.743016 